\title{
Effect of fruit and vegetable intake on oxidative stress and inflammation in COPD: a randomised controlled trial
}

\author{
Francina R. Baldrick*, J. Stuart Elborn\#, , Jayne V. Woodside*, Katherine Treacy\#, \\ Judy M. Bradley ${ }^{\#,+}$, Chris C. Patterson*, Bettina C. Schock", Madeleine Ennis", \\ lan S. Young* and Michelle C. McKinley*
}

ABSTRACT: Epidemiological evidence supports a positive relationship between fruit and vegetable (FV) intake, lung function and chronic obstructive pulmonary disease (COPD). Increasing FV intake may attenuate the oxidative stress and inflammation associated with COPD.

An exploratory randomised controlled trial to examine the effect of increased consumption of FV on oxidative stress and inflammation in moderate-to-severe COPD was conducted. 81 symptomatically stable patients with a habitually low FV intake (two or fewer portions of FV per day) were randomised to the intervention group (five or more portions of FV per day) or the control group (two or fewer portions of FV per day). Each participant received self-selected weekly home deliveries of FV for 12 weeks.

75 participants completed the intervention. There was a significant between-group change in self-reported FV intake and biomarkers of FV intake (zeaxanthin $(p=0.034)$ and $\beta$-cryptoxanthin $(p=0.015))$, indicating good compliance; post-intervention intakes in intervention and control groups were 6.1 and 1.9 portions of FV per day, respectively. There were no significant changes in biomarkers of airway inflammation (interleukin-8 and myeloperoxidase) and systemic inflammation (C-reactive protein) or airway and systemic oxidative stress (8-isoprostane).

This exploratory study demonstrated that patients with moderate-to-severe COPD were able to comply with an intervention to increase FV intake; however, this had no significant effect on airway or systemic oxidative stress and inflammation.

KEYWORDS: Chronic obstructive pulmonary disease, fruit, inflammation, oxidative stress, vegetables

$\mathbf{T}$ he World Health Organization (WHO) have predicted that, between 1990 and 2020, chronic obstructive pulmonary disease (COPD) will move from the sixth- to the third-leading cause of death worldwide [1]. Systemic and airway inflammation and increased levels of oxidative stress are key pathophysiological features of COPD. Thus, factors that can ameliorate these underlying inflammatory processes and/or help to redress the oxidant/ antioxidant balance are of therapeutic interest.

A number of dietary constituents possess antiinflammatory and antioxidant properties and, hence, are of interest with respect to the pathogenesis of COPD. Many cross-sectional studies have demonstrated a significant positive association between fruit and vegetable (FV) intake and forced expiratory volume in $1 \mathrm{~s}$ (FEV1), with evidence particularly strong for fruit $[2,3]$ but also evident for vegetables [4, 5]. Other research has indicated an inverse association between FV intake and COPD symptoms [2, 4], and CAREY et al. [6] demonstrated that a lower fruit intake was associated with a decline in FEV1 over 5-7 yrs of follow-up. Furthermore, fruit intake has also been inversely associated with 20-25-yr incidence of chronic nonspecific lung disease and COPD mortality [2, 7]. A recent systematic review on vitamins and COPD found that the intake of many vitamins commonly found in FV are associated with features of COPD and highlighted the need for prospective randomised controlled trials (RCTs) in this field [8].

It is possible that dietary interventions may attenuate the oxidative stress and inflammation associated with COPD, which may, in turn, translate into beneficial outcomes for patients. Few food-based interventions in COPD have been published to date
AFFILIATIONS

${ }^{\star}$ Centre for Public Health,

"Centre for Infection and Immunity, School of Medicine, Dentistry and Biomedical Sciences, Queen's University Belfast,

${ }^{\text {\#} R e g i o n a l ~ R e s p i r a t o r y ~ C e n t r e, ~ B e l f a s t ~}$ City Hospital, Belfast, and ${ }^{+}$Health and Rehabilitation Sciences Research Institute, University of Ulster, Jordanstown, UK.

\section{CORRESPONDENCE}

M.C. McKinley

Nutrition and Metabolism Group, Centre for Public Health, School of Medicine, Dentistry and Biomedical Sciences, Queen's University Belfast Institute of Clinical Science B (First Floor)

Grosvenor Road

Belfast

BT12 6BJ

UK

E-mail: m.mckinley@qub.ac.uk

Received:

May 202011

Accepted after revision:

Sept 142011

First published online:

Nov 162011 
$[9,10]$. CERDÁ et al. [9] randomised 30 stable COPD patients to consume $400 \mathrm{~mL}$ of pomegranate juice or $400 \mathrm{~mL}$ of a synthetic orange-flavoured drink daily for 5 weeks. They found no significant differences between the groups in lung function or clinical symptoms of COPD. Although juice was provided to participants on a weekly basis, nutritional biomarker compliance data was not reported. To date, there has only been one broad FV intervention in COPD patients. KERANIS et al. [10] reported that a dietary shift from low to modest consumption of FV (based on patients' self-reported intake; objective biomarkers of FV intake were not reported) was associated with an increase in FEV1 in a 3-yr randomised prospective study in COPD patients. Participants were randomised to receive either advice at 6-monthly intervals to increase FV intake $(n=60)$ or to choose their own diet freely $(n=60)$.

Given the epidemiological evidence to date, there is a need to further explore the relationship between FV intake and COPD in RCTs. The aim of this study was to conduct an exploratory RCT to examine the effect of increased consumption of FV on oxidative stress and inflammation in people with moderate-tosevere COPD. The study design incorporated strategies to maximise adherence to the intervention and assessed objective biomarkers of compliance.

\section{METHODS}

\section{Ethics and participants}

Ethical approval was received from The Office for Research Ethics Committees Northern Ireland (Lisburn, UK). All volunteers gave written informed consent. Participants were recruited between February 2007 and February 2009 from respiratory outpatient clinics at four Belfast, UK hospitals. Inclusion criteria were: symptomatically stable, moderate-to-severe COPD (FEV1 $<80 \%$ predicted); and a habitually low FV intake (two or fewer portions of FV per day, assessed by diet history; see later). Exclusion criteria included a history of diabetes mellitus, a regular intake of high-dose antioxidant vitamin supplements and an arterial oxygen tension $<8 \mathrm{kPa}$.

\section{Study design}

This was a 12-week open-label randomised controlled intervention study. A 12-week period was chosen for this study as this has been shown to be sufficient time to ensure an increase in micronutrient status [11] in response to FV interventions, and also to induce changes in plasma C-reactive protein (CRP) and urinary isoprostanes $[12,13]$. Interleukin (IL)- 8 has recently been shown to be responsive to 10-week dietary interventions (with bread [14] and cheese [15]). After obtaining consent, participants were randomly assigned, in blocks of four using a randomnumber generator (www.randomization.com), to the intervention group (five or more portions of FV per day) or the control group (two or fewer portions of FV per day). A portion was as defined by the UK Dept of Health (www.nhs.uk/LiveWell/ 5ADAY), i.e. an $80 \mathrm{~g}$ serving of $\mathrm{FV}$ or $150 \mathrm{~mL}$ of fruit juice. During the 12-week intervention, each participant received selfselected weekly home deliveries of FV from a local retailer. Participants were advised on suitable storage and cooking methods, and how best to incorporate the FV into their diet. Participants were also contacted weekly by the study researcher (F.R. Baldrick) to encourage compliance, record selfreported exacerbations and to discuss any difficulties they were experiencing. Throughout the intervention, participants were advised to maintain their usual level of physical activity and keep other lifestyle factors unchanged.

\section{Study assessments}

Participants attended the Regional Respiratory Centre, Belfast City Hospital, for study assessments at baseline (week 0) and post-intervention (week 12). The same assessments were performed at each visit. A questionnaire was administered to assess alcohol intake and smoking status. Anthropometric measurements (weight, height, and waist and hip circumference) were taken with participants in light indoor clothing and without shoes using standard protocols. A nonfasting blood sample (participants were advised to consume a standard breakfast of tea and toast at home before attending for study assessments), a spot urine sample and an induced sputum sample (see later) were collected. All biological samples were processed and frozen at $-80^{\circ} \mathrm{C}$ within $2 \mathrm{~h}$ of collection. Blood pressure was recorded using a Welch Allyn ${ }_{\mathbb{B}}$ automated sphygmomanometer (Nimed Ltd, Belfast) on the right arm in a seated position after resting for 10-15 min. The average of three readings was recorded. A pulmonary function test, without the use of a bronchodilator, was carried out to assess FEV1 and forced vital capacity according to the American Thoracic Society/European Respiratory Society Task Force recommendations [16]. The predicted references of KNUDSON et al. [17] were used to calculate lung functions. A series of 7 -day diet histories were completed by each participant at weeks 0,6 and 12 in order to assess baseline diet and selfreported compliance with the intervention; average daily FV intake was then hand-counted from these records.

\section{Sputum induction and processing}

$4 \mathrm{~mL}$ of a $4.5 \%$ saline solution was nebulised using an EASYneb II nebuliser (Nebuliser Flaem Nuova S.p.A., San Martino della Battaglia, Italy) by the participant. After $5 \mathrm{~min}$, participants were encouraged to cough and clear a sample. This procedure was repeated until the saline had all been nebulised (approximately 15-20 min). Sputum was processed and treated according to the method of KeLLY et al. [18].

\section{Laboratory analysis}

All laboratory analysis was performed on a blinded basis. Plasma ascorbic acid concentrations were measured on a Cobas FARA centrifugal analyser (Roche, Burgess Hill, UK) with a fluorescent attachment [19]. Serum concentrations of lutein, zeaxanthin, $\beta$-cryptoxanthin, $\alpha$-carotene, $\beta$-carotene and lycopene were determined by reverse-phase high-performance liquid chromatography with diode array detection [20]. Assays were standardised against appropriate National Institute of Standards and Technology reference materials (Gaithersburg, MD, USA). Sputum myeloperoxidase, IL-8 and serum cotinine were analysed by ELISAs on a Triturus ${ }$ EIA Analyzer system (Grifols S.A., Barcelona, Spain). Plasma CRP was measured with standard enzymatic assays (Randox, Crumlin, UK) on an automated ILab-600 biochemical analyser (Instrumentation Laboratories, Warrington, UK). Sputum neutrophil elastase was measured using an in-house continuous activity assay [21]. Sputum and urinary 8-isoprostanes were measured using an AutoDELFIA assay (PerkinElmer, Cambridge, UK) [22]. 


\section{Statistical methods}

All statistical analyses were carried out using the Statistical Package for the Social Sciences (SPSS) for Windows version 17.0 (SPSS Inc, Chicago, IL, USA). Data are presented as mean \pm SD for normally distributed continuous variables. Skewed variables were logarithmically transformed for parametric analysis and presented as geometric mean (interquartile range). An unpaired t-test was used to compare the two groups at baseline and to examine differences in mean change between groups. Within-group changes in end-points were assessed using paired t-tests. Analysis was performed on the intentionto-treat principle; six participants (four in the control group and two in the intervention group) dropped out of the study (reasons given in Results section) and did not attend for the week 12 assessments so could not be included in an intentionto-treat analysis. The Chi-squared test was used to examine differences in nominal or categorical variables between groups.

A secondary analysis was also conducted removing data for participants who had experienced an exacerbation close to completion of the study as this may have had an acute effect on the outcome measures, thus masking the chronic effects of the intervention. Participants were excluded from this analysis if they had an elevated CRP level $\left(>20 \mathrm{mg} \cdot \mathrm{L}^{-1}\right)$ at the end of the study as well as a self-reported exacerbation within the 2 weeks prior to the study end [23]. For the purposes of this study, we defined an exacerbation as "a sustained worsening of respiratory symptoms that is acute in onset and usually requires a patient to seek medical help or alter treatment" [24].

\section{RESULTS}

\section{Summary of participant recruitment}

Participant recruitment is schematised in figure 1.

In total, 454 patients from four hospitals in Belfast were eligible to take part in this study; $373(82.2 \%)$ declined. Reasons for declining participation included being unwilling to give up time or to travel for study assessments $(27.6 \%)$, a dislike of giving blood samples $(4 \%)$, discouragement from other family members $(8 \%)$, feeling too unwell $(47 \%)$ and being uninterested in research $(13.4 \%)$.

A total of 81 participants completed the baseline assessments and 75 participants completed the intervention. There were six dropouts in total following randomisation: four from the control group (one due to back injury, two who were admitted to hospital with other medical complications and one individual was unable to continue to participate owing to personal reasons) and two from the intervention group (one suffered a cardiovascular event and one died due to another medical complication). The intervention was implemented as intended and there were no adverse events associated with the intervention. The availability of paired data is indicated in the tables; it varies for the individual end-points studied according to successful collection of biological material.

\section{Baseline characteristics}

Participants included 46 (57\%) males and 35 (43\%) females. $41 \%$ of participants $(n=33)$ had moderate COPD $(\mathrm{FEV} 1<80 \%$ pred) and 59\% $(n=48)$ had severe COPD (FEV1 $<50 \%$ pred). Two (2.5\%) participants were underweight, 31 (38.3\%) were of normal weight, $20(24.7 \%)$ were overweight and $28(34.5 \%)$

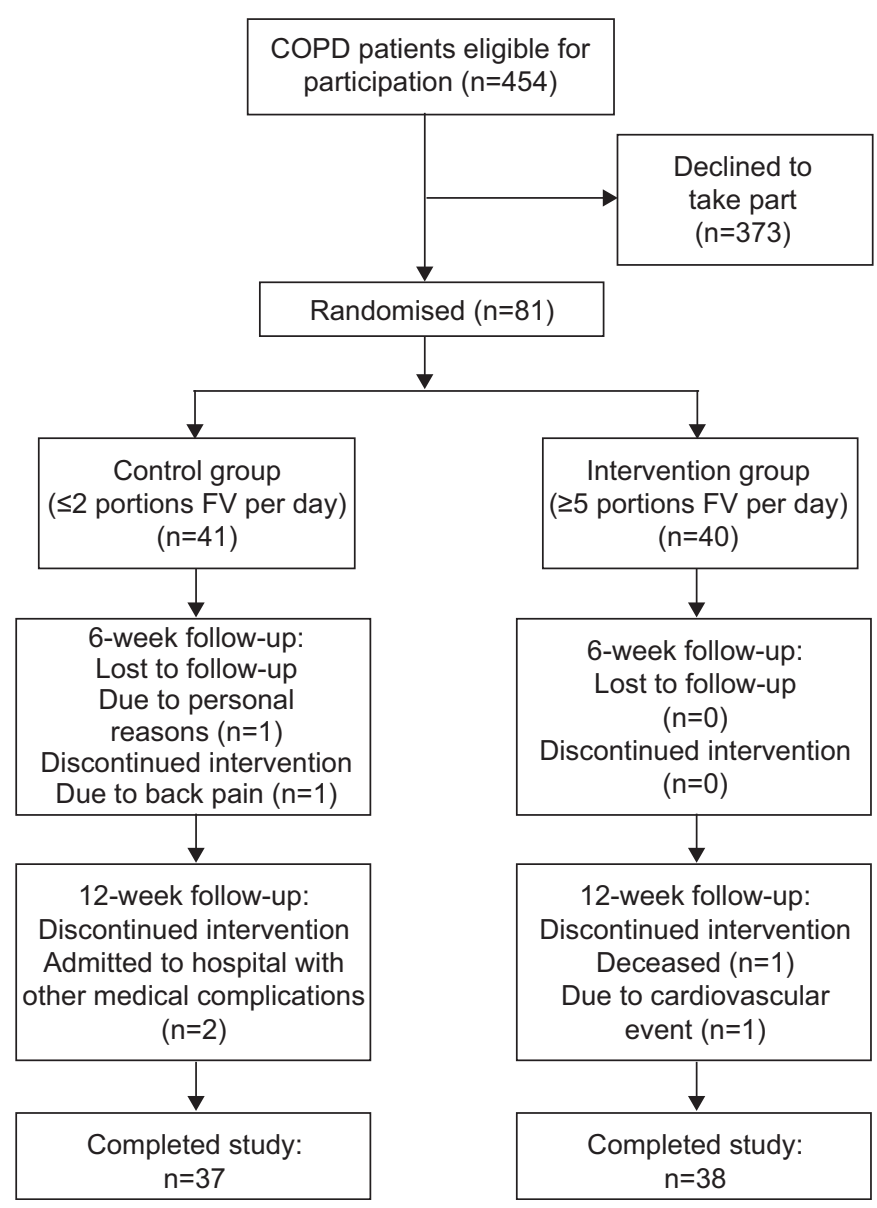

FIGURE 1. Progress of participants through the fruit and vegetable (FV) intervention. COPD: chronic obstructive pulmonary disease

were obese according to $\mathrm{WHO}$ criteria for body mass index. $75 \%$ suffered from comorbidities. There were no significant differences in baseline characteristics between the two randomised groups (table 1). Overall, $94 \%$ of the sample had low serum $\alpha$-carotene status and $93 \%$ had low serum $\beta$-carotene status using cut-offs defined by GEY [25] as being indicative of increased risk of cardiovascular disease and cancer. There was evidence of biochemical depletion of vitamin C (status $<11 \mu \mathrm{mol} \cdot \mathrm{L}^{-1}$ ) in $21 \%$ of the sample (data not shown). There was no significant difference at baseline in inflammatory or oxidative stress biomarkers in participants with moderate versus severe COPD (data not shown).

\section{Change in self-reported FV intake}

Changes in self-reported FV intake are presented in figure 2.

At the end of the study, there was a significant between-group difference $(\mathrm{p}<0.001)$ in change in self-reported FV intake as a result of the intervention; the intervention group increased intake by 4.6 portions per day (from mean \pm SD $1.5 \pm 0.7$ to $6.1 \pm 1.8$ portions per day). The control group also had a marginal increase in FV intake of 0.5 portions per day (from $1.4 \pm 0.6$ to $1.9 \pm 0.5$ portions per day), but were still consuming the target intake for this group of two or fewer portions of FV per day at the end of the intervention. 


\begin{tabular}{|c|c|c|c|}
\hline \multirow{2}{*}{$\begin{array}{l}\text { TABLE } 1 \\
\text { Variable }\end{array}$} & \multicolumn{3}{|c|}{$\begin{array}{l}\text { General baseline characteristics of } 81 \\
\text { participants with moderate-to-severe chronic } \\
\text { obstructive pulmonary disease (COPD) } \\
\text { according to randomisation to a low or high fruit } \\
\text { and vegetable (FV) intake group }\end{array}$} \\
\hline & & Low FV group & High FV group \\
\hline \multicolumn{2}{|l|}{ Participants $\mathbf{n}$} & 41 & 40 \\
\hline \multicolumn{2}{|l|}{ Age yrs } & $61.2 \pm 8.3$ & $63.2 \pm 9.1$ \\
\hline \multicolumn{2}{|l|}{ Males } & $25(61)$ & $21(53)$ \\
\hline \multicolumn{2}{|l|}{ Height $\mathbf{m}$} & $1.66 \pm 0.10$ & $1.66 \pm 0.10$ \\
\hline \multicolumn{2}{|l|}{ Weight kg } & $74.3 \pm 15.5$ & $77.3 \pm 16.7$ \\
\hline \multicolumn{2}{|l|}{$B M I \mathbf{k g} \cdot \mathrm{m}^{-2}$} & $27.2 \pm 5.7$ & $28.2 \pm 6.0$ \\
\hline \multicolumn{2}{|c|}{ Systolic blood pressure $\mathrm{mmHg}$} & $134.7 \pm 20.1$ & $139.6 \pm 19.5$ \\
\hline \multicolumn{2}{|c|}{ Diastolic blood pressure $\mathrm{mmHg}$} & $78.7 \pm 8.8$ & $81.1 \pm 9.7$ \\
\hline \multicolumn{2}{|c|}{ Current smokers } & $22(54)$ & $14(35)$ \\
\hline \multicolumn{2}{|c|}{ Former smokers } & $18(44)$ & $26(65)$ \\
\hline \multicolumn{2}{|c|}{ Smoking history pack-yrs GM (IQR) } & $34.4(20.9-59.9)$ & $40.5(19.9-60.0)$ \\
\hline \multicolumn{2}{|l|}{ Education yrs } & $10.7 \pm 2.1$ & $11.3 \pm 2.2$ \\
\hline \multicolumn{2}{|l|}{ FEV $1 \%$ pred } & $50.4 \pm 17.6$ & $45.2 \pm 17.0$ \\
\hline \multicolumn{2}{|c|}{ Moderate COPD n (FEV $1 \%$ pred range) } & $19(52-85)$ & $14(51-88)$ \\
\hline \multicolumn{2}{|c|}{ Severe COPD n (FEV $1 \%$ pred range) } & $22(20-49)$ & $26(20-50)$ \\
\hline \multicolumn{2}{|l|}{ FVC \% pred } & $77.4 \pm 20.9$ & $73.9 \pm 16.7$ \\
\hline \multicolumn{2}{|c|}{$\mathrm{FEV}_{1 / \mathrm{FVC}} \%$ pred } & $65.6 \pm 16.8$ & $60.4 \pm 15.7$ \\
\hline \multicolumn{2}{|c|}{ FV intake portions per day } & $1.4 \pm 0.6$ & $1.5 \pm 0.7$ \\
\hline
\end{tabular}

Data are presented as mean \pm SD or $n(\%)$, unless otherwise stated. BMI: body mass index; GM: geometric mean; IQR: interquartile range; FEV1: forced expiratory volume in $1 \mathrm{~s}$; \% pred: \% predicted; FVC: forced vital capacity.

\section{Change in biomarkers of FV intake}

Table 2 reports the change in micronutrient status according to study group. There was a significant between-group difference in change in zeaxanthin $(p=0.034)$ and $\beta$-cryptoxanthin $(p=$ $0.015)$ status at 12 weeks. There was also a trend for a betweengroup difference in change in lutein status $(p=0.076)$.

At baseline, $92.5 \%$ of the sample had low serum $\alpha$-carotene status and low serum $\beta$-carotene status using cut-offs defined by GEY [25] as being indicative of increased risk of cardiovascular disease and cancer. At the end of the intervention period, $79 \%$ had low serum $\alpha$-carotene status and $84 \%$ had low serum $\beta$-carotene status. Likewise, for vitamin $C, 12.5 \%$ of the intervention group had evidence of biochemical depletion of vitamin $C$ (status $<11 \mu \mathrm{mol} \cdot \mathrm{L}^{-1}$ ) at baseline compared with $0 \%$ at the end of the intervention.

\section{Change in other lifestyle factors}

There was no significant change in alcohol intake, smoking status or serum cotinine concentrations during the intervention for participants in either the intervention or control group (data not shown).

\section{Change in lung function, oxidative stress and inflammation}

There were no significant differences between the low- and highFV groups for lung function tests or for any of the biomarkers of oxidative stress and inflammation (table 3).

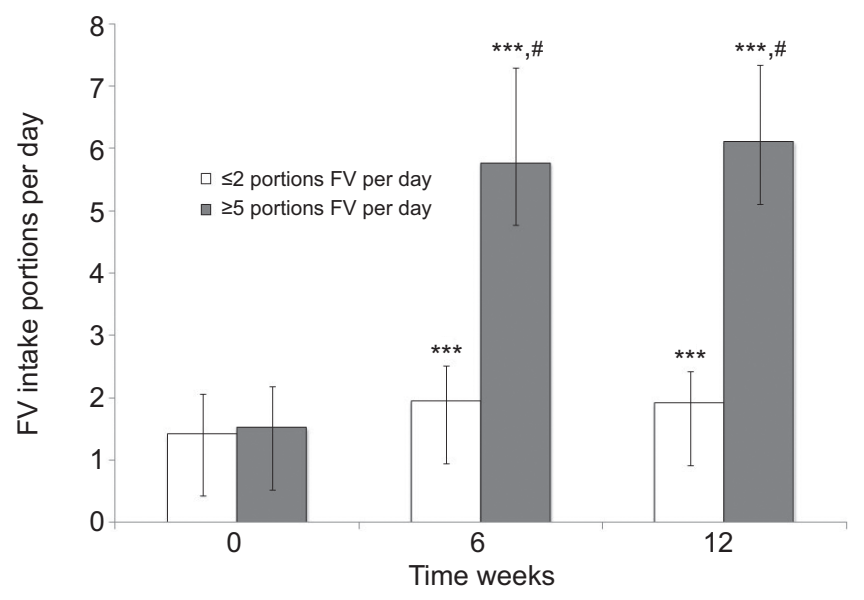

FIGURE 2. Self-reported fruit and vegetable (FV) intake during the intervention period. Data are presented as mean $\pm S D$. ***: $p<0.001$ for within-group comparison by paired t-test; ${ }^{*}: \mathrm{p}<0.001$ for between-group comparison (two or fewer versus five or more portions of FV per day) by unpaired t-test.

\section{Exacerbations during the intervention}

During the 12-week study, 15 participants in the control group had an exacerbation (of which three required hospitalisation) and 20 participants in the intervention group had an exacerbation (of which three required hospitalisation); exacerbation rate did not differ significantly between groups (Chi-squared $p=0.223$ ). In total, 16 participants had a CRP level $>20 \mathrm{mg} \cdot \mathrm{L}^{-1}$ at week 12 and had experienced an exacerbation within the 14 days prior to the end of the study. When the analysis was conducted following removal of these 16 participants, again, there were no significant differences between the two groups for any of the inflammatory or oxidative stress markers measured (data not shown).

\section{DISCUSSION}

The health benefits of FV are well supported by observational epidemiology but it is only recently that data regarding the precise nature of their biological effects has started to emerge from RCTs [26]. Examination of these effects in both healthy and diseased subjects will help to inform both public health messages and evidence-based clinical practice. This trial is the first to explore the effect of increased FV intake on biomarkers of inflammation and oxidative stress in patients with moderate-to-severe COPD. Participant retention rates were high and both self-reported FV intake and biomarkers of FV intake indicated that the intervention was implemented successfully in this group of patients, with the high FV group consuming a mean of six portions of FV per day by the end of the intervention from a baseline of fewer than two portions a day. Despite evidence of good compliance with the study protocol, there was, however, no significant difference between the two groups for markers of oxidative stress or inflammation.

The biomarkers chosen for this study, in particular IL-8, which has been most extensively studied, are reliable indicators of airway inflammation and oxidative stress in COPD populations [27, 28]. IL-8 and systemic CRP correlate positively with disease severity, exacerbation rates and lung function decline in COPD [28]; the lack of a significant difference in disease biomarkers according to disease severity in this study is most likely owing to the large interindividual variability that was 


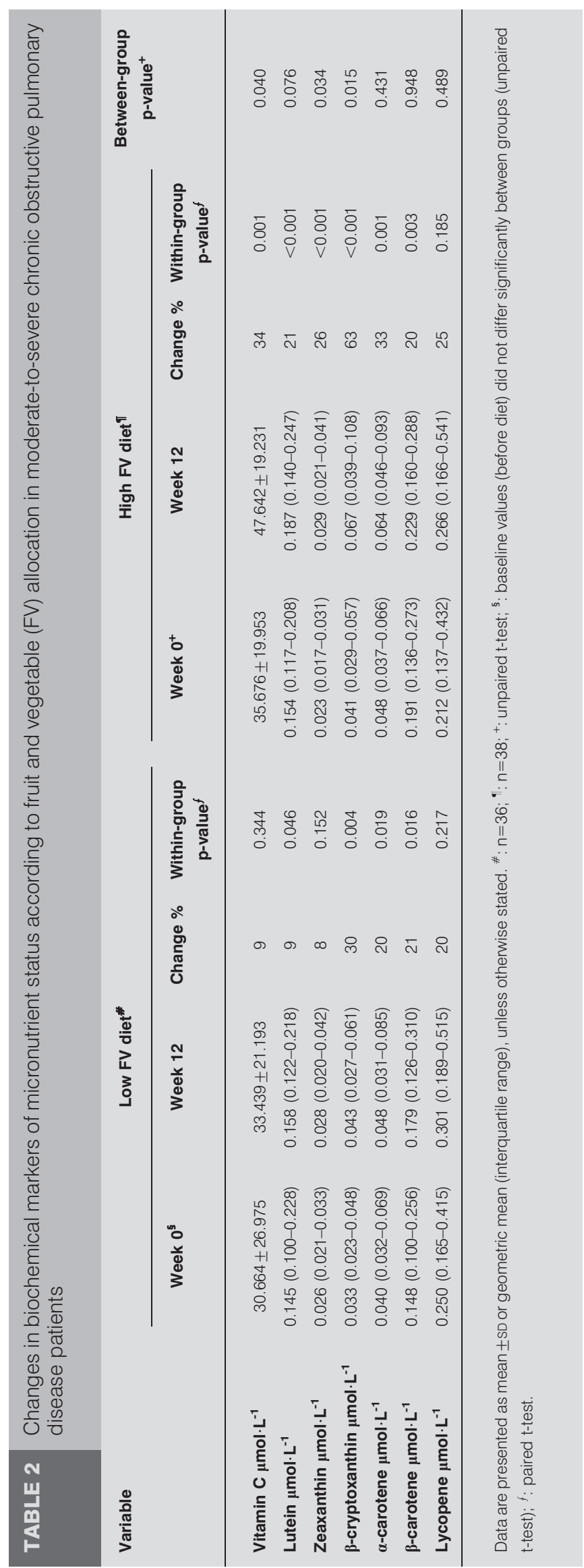

encountered. Recently, food-based interventions with bread $(n=20)$ and cheese $(n=10)[14,15]$ for 10 weeks in generally healthy participants have induced significant lowering of circulating IL-8 concentrations. CRP and isoprostanes are widely regarded as biomarkers of inflammation and oxidative stress, respectively [29, 30]. Crosssectional studies generally support an association between higher FV intake and lower serum levels of CRP; however, FV interventions in healthy populations have shown mixed results [31]. WATZL et al. [12] demonstrated a significant reduction in plasma CRP following 4 weeks on a high-FV diet in healthy nonsmoking males, and a 2-yr Mediterranean-style dietary intervention in patients with metabolic syndrome significantly lowered serum levels of CRP, IL-6, IL-7 and IL-18 [32]. THOMPSON et al. [13] reported a reduction in the excretion of 8-isoprostanes after an 8week high-FV diet in healthy females. However, two recent intervention studies, a 2-month Mediterranean diet intervention [33] and a 2-month FV intervention [26], showed no effect of increased FV intake on circulating CRP, yet these studies were able to demonstrate significant improvements in measures of vascular function (flow-mediated dilatation and venous occlusion plethysmography, respectively), thus indicating that such biomarkers may be dissociated from true biological effects and, therefore, may be of limited use in dietary studies [31]. Further novel biomarkers of COPD may prove to be more sensitive to FV or antioxidant interventions and assessment of a broader panel of biomarkers may be prudent in such work; however, costs often prohibit such explorations.

The assessment of airway inflammation and oxidative stress was limited, to some extent, by the success rate of induced sputum collection, as paired samples were obtained for $77 \%$ of the sample; this should be considered when planning future similar studies. The large intra- and inter-study variability and lack of data on within-subject variability in inflammatory markers in COPD [34] makes it difficult to estimate the sample size for a definitive study; a retrospective power calculation based on the numbers of subjects actually included in the final analysis showed that, assuming no change in end-points in our control group, the study had $>80 \%$ power to detect a $60 \%$ reduction on IL- 8 , a $45 \%$ reduction in myeloperoxidase and a 50\% reduction in plasma CRP in the intervention group. To give an indication of sample sizes required to detect smaller changes, based on the variability of changes in log-transformed IL-8 values observed in our data, a study of 400 COPD subjects (200 subjects per group) would be required to detect a $25 \%$ change in IL- 8 values on intervention with $80 \%$ power. A larger sample size would, of course, have improved the power of the study; however, even trends towards significance were not apparent in the data.

Although circulating antioxidant status increased in this study it cannot be assumed that this, in turn, affected airway antioxidant status. Little is still known about how the antioxidant pool in the respiratory tract lining fluid is maintained and whether or not dietary antioxidant intake can influence this process [35]. A study in nonasthmatic children reported a significant correlation between ascorbate in bronchoalveolar lavage and ascorbate concentrations 


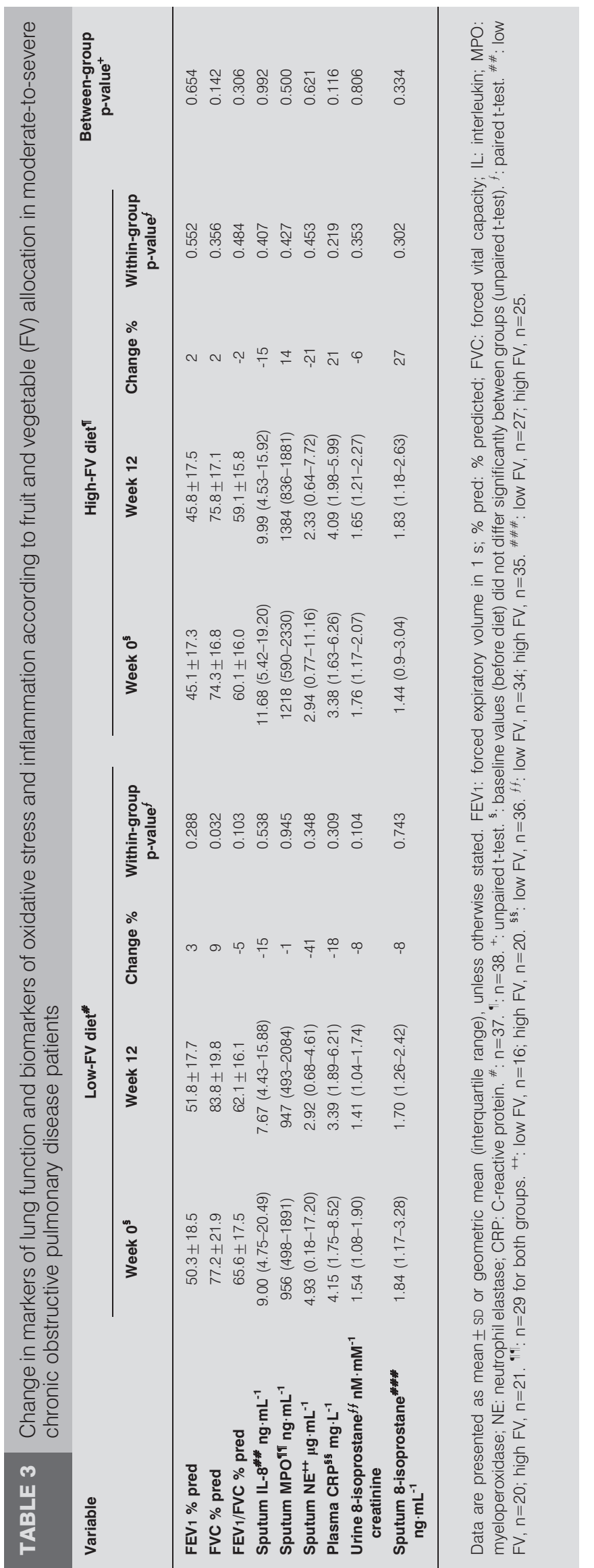

in serum $(r=0.297, p=0.018)$ [36], indicating that dietary antioxidants may be able to influence antioxidant defences in the lung. An investigation of antioxidant levels in induced sputum samples from this study would help to elucidate the relationship between dietary antioxidant intake and antioxidant concentrations within the respiratory tract.

To our knowledge, there is only one other FV intervention in COPD patients to date. KERANIS et al. [10] recently reported findings from a 3-yr prospective study in which participants from COPD outpatient clinics in Greece were randomised to consume a diet with increased consumption of fresh FV $(n=60)$ or to consume a diet of their own free choice $(n=60)$. The intervention group was given advice to consume FV that was reinforced every 6 months at scheduled outpatient appointments. The study reported that the intervention group, who changed their diet from low to modest consumption of FV (change in terms of portions for fruit and vegetables not reported), displayed an increase in FEV1, whereas controlgroup patients exhibited a decline in lung function $(p=0.03$ for difference between groups). The lack of objective compliance data in the long-term study by KERANIS et al. [10] makes it difficult to confidently attribute the positive observations to increased consumption of FV. KERANIS et al. [10] did, however, employ a long-term follow-up of participants and it is possible that, given the chronic nature of COPD, such a lengthy followup may be necessary in order to examine the true biological effects of dietary interventions in this population. It is also encouraging that the patient group studied by KERANIS et al. [10], $\sim 78 \%$ of whom had moderate-to-severe COPD, were able to increase FV intake and sustain this for 3 yrs; however, the caveat regarding objective biomarkers of FV intake discussed above remains a limiting issue.

In the present study, there was a significant change in FV intake between the intervention and control group (difference in change in FV intake was 4.2 portions per day between the groups), and this was reflected in significant between-group differences in biomarkers of FV intake (zeaxanthin and $\beta$ cryptoxanthin). The control group did increase their intake of FV by, on average, 0.5 portions per day but their overall mean intake remained within the study target of two or fewer portions per day. This increase in intake in the control group was minimal and not unexpected given the fact that the intervention involved home delivery of FV to both groups, as well as weekly contact with the study researcher, both of which would heighten awareness of consumption.

The limitations of the present study must be acknowledged. This was, by necessity, an open-label study; however, the laboratory analysis was performed on a blinded basis. It was also a shortterm intervention and so results cannot be extrapolated to the situation of chronic consumption. Furthermore, two barriers (cost and access) to FV intake were removed in the study in order to enhance compliance. Participants were asked not to make any other lifestyle changes during the study and they reported no change in smoking behaviour or alcohol intake. However, physical activity was not assessed pre- and post-intervention. While changes in physical activity may be unlikely in this population, it cannot be ruled out as a potential confounder. Exacerbation assessment is also a limiting factor. Although exacerbation rate did not appear to differ between groups, it is 
important to note that exacerbations were self-reported and were not verified by medical records. Finally, the participants in this study may not be representative of all patients with moderate-to-severe COPD, $82 \%$ of eligible participants declined to participate and patients with lower body mass indices were under-represented; it is likely that such individuals declined to participate owing to the perceived demands of the study.

Further work in this area should not be precluded based on the results of this initial study alone as many individual factors could affect the outcome of such work. Dietary interventions such as this may have differing effects in different subgroups of COPD patients. The study duration and end-points that are most likely to be responsive are also a critical consideration; it is possible that longer-term dietary interventions may be more effective in COPD. In terms of the nature of the intervention itself, there are many possible approaches, each with their own merits; for example, one could hypothesise that a broader dietary approach that focuses on several key foods or food groups, rather than focusing on one particular food group (FV), may be more likely to have a beneficial effect.

In conclusion, this study demonstrated that patients with moderate-to-severe COPD were able to comply with an intervention to increase FV intake; however, this increased intake had no significant effect on airway or systemic oxidative stress and inflammation. Although no signal was apparent, a potentially beneficial effect of increased FV intake in COPD cannot be excluded based on this exploratory study alone, as longer-term interventions with different end-points may be required to demonstrate biological effects in this population. The experiences from this trial in terms of feasibility of recruitment, challenges of induced sputum sample collection and variance in the study end-points should be considered when planning future studies in this area.

\section{SUPPORT STATEMENT}

This study was funded by Northern Ireland Chest, Heart and Stroke (NICHS)

\section{CLINICAL TRIAL}

This study is registered at www.clinicaltrials.gov with identifier number NCT00435708.

\section{STATEMENT OF INTEREST}

Statements of interest for C.C. Patterson, I.S. Young and for the study itself can be found at www.erj.ersjournals.com/site/misc/statements. xhtml

\section{ACKNOWLEDGEMENTS}

The authors would like to acknowledge the participants that kindly volunteered to take part in this study and the nursing staff of the Regional Respiratory Centre, Belfast City Hospital, (Belfast, UK) for their contribution to this trial. We are grateful to C. McMaster, C. Mercer and S. Gilchrist (School of Medicine, Dentistry and Biomedical Sciences, Queen's University Belfast) for carrying out inflammatory marker and nutritional biomarker assays, and to Unilever Research (Colworth, UK) for carrying out the 8-isoprostane assays.

\section{REFERENCES}

1 Lopez AD, Murray CC. The global burden of disease, 1990-2020. Nat Med 1998; 4: 1241-1243.
2 Smit HA, Grievink L, Tabak C. Dietary influences on chronic obstructive lung disease and asthma: a review of the epidemiological evidence. Proc Nutr Soc 1999; 58: 309-319.

3 Romieu I, Trenga C. Diet and obstructive lung diseases. Epidemiol Rev 2001; 23: 268-287.

4 Tabak C, Smit HA, Heederik D, et al. Diet and chronic obstructive pulmonary disease: independent beneficial effects of fruits, whole grains, and alcohol (the MORGEN study). Clin Exp Allergy 2001; 31: 747-755.

5 Kelly Y, Sacker A, Marmot M. Nutrition and respiratory health in adults: findings from the Health Survey for Scotland. Eur Respir J 2003; 21: 664-671.

6 Carey IM, Strachan DP, Cook DG. Effects of changes in fresh fruit consumption on ventilatory function in healthy British adults. Am J Respir Crit Care Med 1998; 158: 728-733.

7 Walda IC, Tabak C, Smit HA, et al. Diet and 20-year chronic obstructive pulmonary disease mortality in middle-aged men from three European countries. Eur J Clin Nutr 2002; 56: 638-643.

8 Tsiligianni IG, van der Molen T. A systematic review of the role of vitamin insufficiencies and supplementation in COPD. Respir Res 2010; 11: 171.

9 Cerdá B, Soto C, Albaladejo MD, et al. Pomegranate juice supplementation in chronic obstructive pulmonary disease: a 5week randomized, double-blind, placebo-controlled trial. Eur J Clin Nutr 2006; 60: 245-253.

10 Keranis E, Makris D, Rodopoulou P, et al. Impact of dietary shift to higher-antioxidant foods in COPD: a randomised trial. Eur Respir J 2010; 36: 774-780.

11 Baldrick FR, Woodside JV, Elborn JS, et al. Biomarkers of fruit and vegetable intake in human intervention studies: a systematic review. Crit Rev Food Sci Nutr 2011; 51: 795-815.

12 Watzl B, Kulling SE, Moseneder J, et al. A 4-wk intervention with high intake of carotenoid-rich vegetables and fruit reduces plasma C-reactive protein in healthy, nonsmoking men. Am J Clin Nutr 2005; 82: 1052-1058.

13 Thompson HJ, Heimendinger J, Sedlacek S, et al. 8-Isoprostane $F_{2 \alpha}$ excretion is reduced in women by increased vegetable and fruit intake. Am J Clin Nutr 2005; 82: 768-776.

14 Sofi F, Ghiselli L, Cesari F, et al. Effects of short-term consumption of bread obtained by an old Italian grain variety on lipid, inflammatory, and hemorheological variables: an intervention study. J Med Food 2010; 13: 615-620.

15 Sofi F, Buccioni A, Cesari F, et al. Effects of a dairy product (pecorino cheese) naturally rich in cis-9, trans-11 conjugated linoleic acid on lipid, inflammatory and haemorheological variables: a dietary intervention study. Nutr Metab Cardiovasc Dis 2010; 20: 117-124.

16 Miller MR, Hankinson J, Brusasco V, et al. Standardisation of spirometry. Eur Respir J 2005; 26: 319-338.

17 Knudson RJ, Lebowitz MD, Holberg CJ, et al. Changes in the normal maximal expiratory flow-volume curve with growth and aging. Am Rev Respir Dis 1983; 127: 725-734.

18 Kelly MG, Brown V, Martin SL, et al. Comparison of sputum induction using high-output and low-output ultrasonic nebulizers in normal subjects and patients with COPD. Chest 2002; 122: 955-959.

19 Vuilleumier JP, Keck E. Fluorometric assay of vitamin C in biological materials using a centrifugal analyser with fluorescence attachment. J Micronutr Anal 1989; 5: 25-34.

20 Craft NE. Carotenoid reversed-phase high-performance liquid chromatography methods: reference compendium. Methods Enzymol 1992; 213: 185-205.

21 Martin SL, Moffitt KL, McDowell A, et al. Association of airway cathepsin B and $\mathrm{S}$ with inflammation in cystic fibrosis. Pediatr Pulmonol 2010; 45: 860-868.

22 Bailey DM, Williams C, Betts JA, et al. Oxidative stress, inflammation and recovery of muscle function after damaging exercise: effect 
of 6-week mixed antioxidant supplementation. Eur J Appl Physiol 2011; 111: 925-936.

23 Wedzicha JA, Seemungal TA. COPD exacerbations: defining their cause and prevention. Lancet 2007; 370: 786-796.

24 Pauwels R, Calverley P, Buist AS, et al. COPD exacerbations: the importance of a standard definition. Respir Med 2002; 98: 99-107.

25 Gey KF. Vitamins E plus C and interacting conutrients required for optimal health. A critical and constructive review of epidemiology and supplementation data regarding cardiovascular disease and cancer. Biofactors 1998; 7: 113-174.

26 McCall DO, McGartland CP, McKinley MC, et al. Dietary intake of fruits and vegetables improves microvascular function in hypertensive subjects in a dose-dependent manner. Circulation 2009; 119: 2153-2160.

27 Cazzola M, MacNee W, Martinez FJ, et al. Outcomes for COPD pharmacological trials: from lung function to biomarkers. Eur Respir J 2008; 31: 416-468.

28 Stockley RA. Progression of chronic obstructive pulmonary disease: impact of inflammation, comorbidities and therapeutic intervention. Curr Med Res Opin 2009; 25: 1235-1245.

29 Gan WQ, Man SF, Senthilselvan A, et al. Association between chronic obstructive pulmonary disease and systemic inflammation: a systematic review and a meta-analysis. Thorax 2004; 59: 574-580.
30 Louhelainen N, Myllarniemi M, Rahman I, et al. Airway biomarkers of the oxidant burden in asthma and chronic obstructive pulmonary disease: current and future perspectives. Int J Chron Obstruct Pulmon Dis 2008; 3: 585-603.

31 McCall DO, McGartland CP, McKinley MC, et al. The effect of increased dietary fruit and vegetable consumption on endothelial activation, inflammation and oxidative stress in hypertensive volunteers. Nutr Metab Cardiovasc Dis 2011; 21: 658-664.

32 Esposito K, Marfella R, Ciotola M, et al. Effect of a Mediterranean-style diet on endothelial dysfunction and markers of vascular inflammation in the metabolic syndrome. JAMA 2004; 292: 1440-1446.

33 Rallidis LS, Lekakis J, Kolomvotsou A, et al. Close adherence to a Mediterranean diet improves endothelial function in subjects with abdominal obesity. Am J Clin Nutr 2009; 90: 263-268.

34 Barnes PJ, Chowdhury B, Kharitonov SA, et al. Pulmonary biomarkers in chronic obstructive pulmonary disease. Am J Respir Crit Care Med 2006; 174: 6-14.

35 Kelly FJ. Vitamins and respiratory disease: antioxidant micronutrients in pulmonary health and disease. Proc Nutr Soc 2005; 64: 510-526.

36 Schock BC, Young IS, Brown V, et al. Antioxidants and protein carbonyls in bronchoalveolar lavage fluid of children: normal data. Pediatr Res 2001; 49: 155-161. 